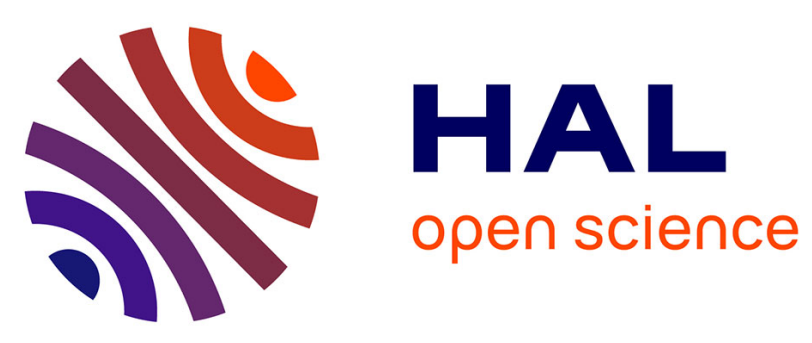

\title{
A Unified Mobile Manipulator Control for On-line Tip-over Avoidance Based on ZMP Disturbance Observer*
}

Kamal Mohy El Dine, Juan Antonio Corrales Ramon, Youcef Mezouar, Jean-Christophe Fauroux

\section{To cite this version:}

Kamal Mohy El Dine, Juan Antonio Corrales Ramon, Youcef Mezouar, Jean-Christophe Fauroux. A Unified Mobile Manipulator Control for On-line Tip-over Avoidance Based on ZMP Disturbance Observer*. 2018 IEEE International Conference on Robotics and Biomimetics (ROBIO), Dec 2018, Kuala Lumpur, Malaysia. pp.1437-1443, 10.1109/ROBIO.2018.8665042 . hal-02098629

\section{HAL Id: hal-02098629 \\ https://hal.uca.fr/hal-02098629}

Submitted on 12 Apr 2019

HAL is a multi-disciplinary open access archive for the deposit and dissemination of scientific research documents, whether they are published or not. The documents may come from teaching and research institutions in France or abroad, or from public or private research centers.
L'archive ouverte pluridisciplinaire HAL, est destinée au dépôt et à la diffusion de documents scientifiques de niveau recherche, publiés ou non, émanant des établissements d'enseignement et de recherche français ou étrangers, des laboratoires publics ou privés. 


\title{
A Unified Mobile Manipulator Control for On-line Tip-over Avoidance Based on ZMP Disturbance Observer*
}

\author{
Kamal Mohy El Dine, Juan-Antonio Corrales-Ramon, Youcef Mezouar and Jean-Christophe Fauroux
}

\begin{abstract}
Introducing mobile manipulators into large working areas has gained importance in the last years. This is due to the fact that manipulators mounted on vehicles have many merits over the ones with fixed bases. The extended workspace and the maneuverability are main advantages of mobile manipulators. However, stability of the system is critical and closely related to the vehicle's motion, arm's motion and the disturbances which may cause the system to tumble. To address the above issue, this paper presents a novel unified dynamic control that keeps the zero moment point (ZMP) on a desired stable position without affecting the end-effector's motion. A redundant scheme is used to develop the motion controller and a novel ZMP disturbance observer is derived to estimate the modeling and inertial errors of the zero moment point to be controlled. Finally, the performance of the proposed controller is validated through dynamic simulation on a 7-dof anthropomorphic arm fixed on differential mobile base. The overall controller not only ensures a good ZMP control for stability but it is also devised to control the end-effector to follow accurately a desired spatial trajectory.
\end{abstract}

\section{INTRODUCTION}

Due to their significant manipulability and large workspace, mobile manipulators have attracted the interest of researchers in last years. The system generally consists of a mobile base with a fixed robotic arm on its top. Such systems are being widely deployed in industries and risky tasks such as fire fighting and transportation of toxic materials like asbestos and nuclear wastes [1], [2]. Besides, the stability of mobile manipulators with relatively high center of mass (COM) and small size support is critical. It limits their efficiency and productivity as they might tip over. For the various applications of mobile manipulators, several stability strategies have been considered, they can be classified in three categories, namely: (1) stability monitoring and evaluation through static and dynamic indices, (2) offline planning based on stability constraints and (3) on-line stability control. In the first category, a vast research has been done. The shortest horizontal distance between the COM and the support pattern boundary projected onto a horizontal plane was used as static stability index in [3]-[5]. Other static approaches like the gradient based method and the potential energy criterion were proposed to evaluate stability

\footnotetext{
*The research leading to these results has received funding from the European Union's Horizon 2020 research and innovation programme under grant agreement No. 687593.

*The author would like to thank Miguel ARANDA for his valuable comments and discussions.

The authors are with Institut Pascal, SIGMA Clermont, Université Clermont Auvergne, CNRS, F-63000 Clermont-Ferrand, $\quad$ France. $\quad$ Kamal.Mohy_El_Dine, Juan-Antonio.Corrales-Ramon, Youcef.Mezouar, Jean-Christophe.Fauroux\}@sigma-clermont.fr
}

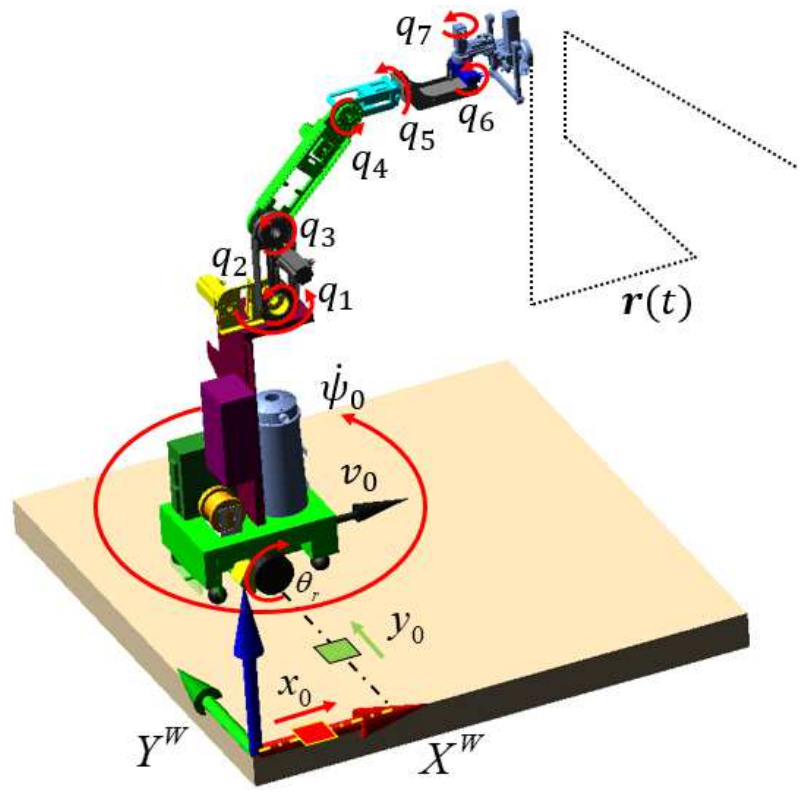

Fig. 1: A top heavy mobile manipulator following a spatial trajectory $\boldsymbol{r}(t)$ is critically unstable and might tip over. The kinematics of the robot are depicted in the figure above.

in [6], [7], then the use of projection plane was eliminated using a net force vector in [8]. However, static approaches were not efficient for top-heavy systems with high dynamics and the zero moment point was introduced for the first time in the context of mobile manipulators in [9]. The authors of [10]-[12] have considered forces and system dynamics, they introduced them to the criteria proposed in [4], [6], [7] respectively. Evaluation of the previous approaches was presented in [13] and a new tip-over stability measure based on moments was derived. Although it is true that the indices in category (1) were able to detect falling, regardless of their accuracy, they are not sufficient to recover or avoid tumbling when the system dynamics are significant. To deal with this issue, researchers have been studying motion planning for mobile manipulators considering stability (category (2)). A plan that permits a mobile manipulator to execute quick tasks considering the dynamic limits of the vehicle is presented in [14], redundancy was used for optimal task planning and stability [15]. In [16]-[18], ZMP was used to plan motions based on gradient methods and inertias, restraints on the end-effectors were not considered. Conventional methods developed in category (1) were used to plan stable motions in [12], then stability was evaluated 
using the forces and moments acting on the line connecting two contact points. More research that studied the stability of mobile manipulators during the planning stage is presented in [19], [20]. Yet, off-line motion planning and optimization processes are time consuming, they can not handle general situations and overcome disturbances. When the end-effector motion is subject to disturbances or it is driven based on a sensor, some reactions need to be done in real time to compensate instability. Finally, in category (3), many works benefit from the base/arm motions to compensate the stability on-line. In [3], [21] the center of gravity is used to optimize and control stability, but the dynamics were ignored. In [22]-[24] the vehicle-arm motion coordination is based on potential functions and the valid stable regions are based on (ZMP) criterion. However, these methods are weak in treating dynamic environments and have less efficiency in moving the robot as one synchronized unit. The arm motions were used for stability compensation in [25], [26], they showed good performance but there is no restriction on the behaviors of the end-effector. Works that deal with online stability control using fuzzy logic, neural networks and quadratic programming were presented in [27]-[29]. On-line stability control challenges come from the dynamic coupling between the mobile base and the arm on one hand, and the nonholonomic constraints of the base on the other. These two issues have been taken into consideration in the literature of mobile manipulators control. Decentralized control for base and arm have been developed in [30]-[33]. Although the interaction between the two controllers is considered, it still difficult to tune [34], [35]. On the contrary, centralized controllers deal with the robot as one system [36]-[39]. To the best of our knowledge, a unified dynamic scheme that actively controls the stability of the whole system on a desired point has not been considered in the literature of mobile manipulators. Additionally, unlike previous methods that use ZMP and ignore the errors in COM positions of the links and their inertia, the ZMP reference used in our controller is based on disturbance observer that estimates the effect of the mentioned errors. Our method does not need planning, it is based on real time adaptive control to damp the oscillatory motions. Feed-forward terms are used to track the desired stable point on the ground to prevent the robot from tipping over. The end-effector constraints to follow a desired spatial trajectory are retained at the same time.

\section{CONTROL FRAMEWORK}

\section{A. Operational Space Dynamics}

To develop a unified kinematic and dynamic models for the whole system, the mobile base is considered as a set of virtual joints and modeled along with the manipulator (Figure 1). The operational space equations of a robot with $m$-dof executing a general task of $n$-dof are:

$$
\boldsymbol{h}_{c}=\boldsymbol{\Lambda}(\boldsymbol{q}) \boldsymbol{\alpha}_{c}+\boldsymbol{\mu}(\boldsymbol{q}, \dot{\boldsymbol{q}})
$$

$\boldsymbol{q}=\left[\boldsymbol{q}_{b}, \boldsymbol{q}_{a}\right]^{T}$ is the $m$ joint positions. $\boldsymbol{q}_{b}$ is the base virtual joints $\left[x_{0}, y_{0}, \psi_{0}\right]^{T}$, and $\boldsymbol{q}_{a}$ is the arm joint positions $\left[q_{1} \ldots q_{m-3}\right]^{T} . \boldsymbol{h}_{c} \in R^{n}$ is the generalized end-effector wrench [40], $\boldsymbol{\alpha}_{c}$ is the acceleration command . $\boldsymbol{\mu}(\boldsymbol{q}, \dot{\boldsymbol{q}})$ is the $(n \times 1)$ function to compensate for Coriolis, gravitational and friction forces in the workspace. $\boldsymbol{\Lambda}(\boldsymbol{q})$ is the pseudo-inertia matrix defined by:

$$
\boldsymbol{\Lambda}(\boldsymbol{q})=\left(\boldsymbol{J}(\boldsymbol{q}) \boldsymbol{A}(\boldsymbol{q})^{-1} \boldsymbol{J}^{T}(\boldsymbol{q})\right)^{-1}
$$

with $\boldsymbol{J}(\boldsymbol{q})$ denoting the $(n \times m)$ kinematic Jacobian matrix, $\boldsymbol{A}(\boldsymbol{q})$ is the full rank $(m \times m)$ robot inertia matrix. Finally, for a redundant system with $m>n$ the joint forces $\tau_{c}=$ $\left[\boldsymbol{\tau}_{b}, \boldsymbol{\tau}_{a}\right]^{T}$ corresponding to $\boldsymbol{h}_{c}$ are:

$$
\boldsymbol{\tau}_{c}=\boldsymbol{J}^{T}(\boldsymbol{q}) \boldsymbol{h}_{c}+\left(\boldsymbol{I}-\boldsymbol{J}^{T}(\boldsymbol{q}) \tilde{\boldsymbol{J}}^{T}(\boldsymbol{q})\right) \boldsymbol{\tau}_{n u l l}+\boldsymbol{\tau}_{\text {dis }}
$$

$\tilde{\boldsymbol{J}}(\boldsymbol{q})$ is the dynamically consistent generalized inverse:

$$
\tilde{\boldsymbol{J}}(\boldsymbol{q})=\boldsymbol{A}^{-1}(\boldsymbol{q}) \boldsymbol{J}^{T}(\boldsymbol{q}) \boldsymbol{\Lambda}(\boldsymbol{q})
$$

$\tau_{n u l l}$ are joint forces that can be applied in the null space without affecting the end-effector's dynamic behavior. In case $n>=m$, the system is not redundant and the second term in eq.(3) will be canceled. $\tau_{d i s}$ is the disturbance torque on the joints:

$$
\boldsymbol{\tau}_{\text {dis }}=\boldsymbol{\tau}_{\text {int }}+\boldsymbol{\tau}_{\text {ext }}+\boldsymbol{\tau}_{\text {fri }}+\boldsymbol{\tau}_{\text {unc }}
$$

The disturbance torques $\boldsymbol{\tau}_{\text {int }}, \boldsymbol{\tau}_{\text {ext }}, \boldsymbol{\tau}_{\text {fri }}$ and $\boldsymbol{\tau}_{\text {unc }}$ are the internal, external, friction and uncertain motor dynamics respectively. If the base is subject nonholonomic constraints, its corresponding virtual joint forces, $\boldsymbol{\tau}_{b}=\left[f_{x 0}, f_{y 0}, \tau_{z 0}\right]^{T}$ needs to be transposed into the differential wheels torque $\tau_{w}$ as:

$$
\boldsymbol{\tau}_{w}=\boldsymbol{J}_{d}^{+} \boldsymbol{\tau}_{b}
$$

$\boldsymbol{J}_{d}$ is a dynamic Jacobian that takes into account the nonholonomic constraints and $\boldsymbol{J}_{d}^{+}$is its pseudo inverse, they are dependent on the mobile base type. For differential drive robots (ex: Figure 1) they can be expressed as:

$$
\begin{gathered}
\boldsymbol{J}_{d}=\left[\begin{array}{cc}
\frac{1}{R} \cos \psi_{0} & \frac{1}{R} \cos \psi_{0} \\
\frac{1}{R} \sin \psi_{0} & \frac{1}{R} \sin \psi_{0} \\
\frac{W}{2 R} & \frac{-W}{2 R}
\end{array}\right] \\
\boldsymbol{J}_{d}^{+}=\left[\begin{array}{lll}
\frac{R \cos \psi_{0}}{2} & \frac{R \sin \psi_{0}}{2} & \frac{R}{W} \\
\frac{R \cos \psi_{0}}{2} & \frac{R \sin \psi_{0}}{2} & -\frac{R}{W}
\end{array}\right]
\end{gathered}
$$

$W, R$ and $\psi_{0}$ are the mobile base parameters defined in Table I.

\section{B. Zero Moment Point Disturbance Observer}

The ZMP is an important concept of dynamic stability. In the literature mentioned previously, it is used in its original form:

$$
\begin{aligned}
& Z M P_{x}=\frac{\Sigma_{i} m_{i} x_{i}\left(\ddot{z}_{i}+g\right)-\Sigma_{i} m_{i} \ddot{x}_{i} z_{i}-\Sigma_{i} I_{i y} \ddot{\theta}_{i y}}{\Sigma_{i} m_{i}\left(\ddot{z}_{i}+g\right)} \\
& Z M P_{y}=\frac{\Sigma_{i} m_{i} y_{i}\left(\ddot{z}_{i}+g\right)-\Sigma_{i} m_{i} \ddot{y}_{i} z_{i}+\Sigma_{i} I_{i x} \ddot{\theta}_{i x}}{\Sigma_{i} m_{i}\left(\ddot{z}_{i}+g\right)}
\end{aligned}
$$

Where $m_{i}$ is the mass of ith body; $x_{i}, y_{i}$ and $z_{i}$ are the positions of centers of mass of each body segment; $I_{i x}$ And $I_{i y}$ are the inertia around $x$ and $y$ respectively; $\ddot{\theta}_{i} x$ and $\ddot{\theta}_{i} y$ are the angular accelerations of each link around $x$ and 


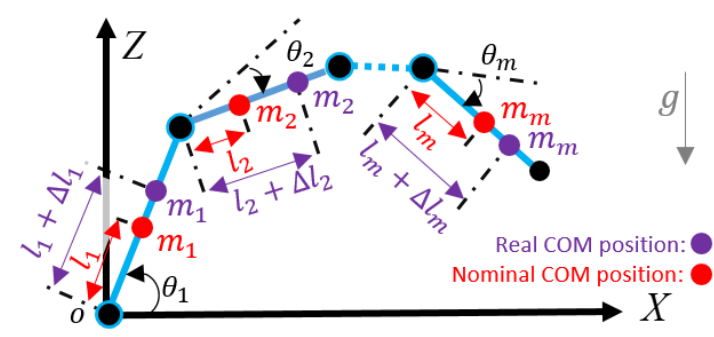

Fig. 2: COM position errors illustrated on a $m$-link robot.

$y$ respectively. In (9) and (10) the inertias are assumed to be nominal, and the COM position of each link assumed to be known. Nevertheless, in practice it is not the case. This point can be illustrated with the $m$-link manipulator in Figure 2. The Errors in the center of mass positions $\Delta l=$ $\left[\Delta l_{1}, \Delta l_{2}, \cdots, \Delta l_{m}\right]$ have a significant effect on $Z M P_{x}$ (see eq.(9)). Inertial errors will also have notable effects on stability, especially when the mobile base is relatively small and the robot is top heavy (Figure 1). The dynamics of $m$ links can be expressed as:

$$
\boldsymbol{\tau}_{\text {mlink }}=\boldsymbol{M}_{n}(\boldsymbol{\theta}) \ddot{\boldsymbol{\theta}}+\boldsymbol{\tau}_{\text {dis }}
$$

$\boldsymbol{\theta}=\left[\theta_{1}, \theta_{2}, \cdots, \theta_{m}\right]$ is the joints value vector, $\boldsymbol{\tau}_{\text {mlink }}=$ $\left[\tau_{\text {mlink }}^{1}, \tau_{\text {mlink }}^{2}, \cdots, \tau_{\text {mlink }}^{m}\right]$ is the joints torque value , $\boldsymbol{M}(\boldsymbol{\theta})$ is the inertia matrix and $\boldsymbol{M}_{n}(\boldsymbol{\theta})$ is the nominal one. Reformulation of eq.(5) is presented in [41], [42]. Uncertain motor dynamics are considered small enough to be ignored, and $\boldsymbol{\tau}_{\text {dis }}$ is estimated using disturbance observer (DOB) [41] as:

$$
\hat{\boldsymbol{\tau}}_{d i s}=\boldsymbol{\tau}_{\text {int }}+\boldsymbol{\tau}_{\text {ext }}+\boldsymbol{\tau}_{f r i}+\left(\boldsymbol{M}-\boldsymbol{M}_{n}\right) \ddot{\boldsymbol{\theta}}
$$

$\boldsymbol{\tau}_{\text {ext }}=0$ when there is no contact with the environment. $\boldsymbol{\tau}_{\text {int }}$ is equivalent to gravity and Coriolis torques in joint space $\boldsymbol{H}(\boldsymbol{\theta}, \ddot{\boldsymbol{\theta}})$. The errors $\Delta l$ will result internal torque disturbances. Eq.(12) can be rewritten as:

$$
\hat{\boldsymbol{\tau}}_{d i s}=\boldsymbol{H}_{n}+\boldsymbol{\tau}^{\left(\boldsymbol{H}-\boldsymbol{H}_{n}\right)}+\boldsymbol{\tau}_{f r i}+\left(\boldsymbol{M}-\boldsymbol{M}_{n}\right) \ddot{\boldsymbol{\theta}}
$$

$\boldsymbol{\tau}\left(\boldsymbol{H}-\boldsymbol{H}_{n}\right)$ is the disturbance torque corresponding to the COMs position errors. $\boldsymbol{H}_{n}$ is the nominal gravity and Coriolis torques in joint space, it can be easily calculated using the nominal distances and dynamic parameters. $\boldsymbol{\tau}_{\text {fri }}$ is obtained by friction identification tests. Hence, the resultant disturbance from COM position errors and inertial errors will be:

$$
\hat{\boldsymbol{\tau}}_{d i s}=\boldsymbol{\tau}^{\left(\boldsymbol{H}-\boldsymbol{H}_{n}\right)}+\left(\boldsymbol{M}-\boldsymbol{M}_{n}\right) \ddot{\boldsymbol{\theta}}
$$

This disturbance torque can be projected to the workspace forces using the Jacobian of the $m$-link manipulator $\boldsymbol{J}_{\text {mlink }}$ :

$$
\hat{\boldsymbol{h}}_{\text {dis }}=\left(\boldsymbol{J}_{\text {mlink }}^{T}\right)^{-1} \hat{\boldsymbol{\tau}}_{\text {dis }}
$$

For a general task of dimension $n \in R^{6}, \hat{\boldsymbol{h}}_{\text {dis }}=$ $\left[\hat{\boldsymbol{f}}_{d i s}, \hat{\boldsymbol{m}}_{d i s}\right]^{T}$, where $\hat{\boldsymbol{f}}_{\text {dis }}=\left[\hat{f}_{d i s}^{x}, \hat{f}_{d i s}^{y}, \hat{f}_{d i s}^{z}\right]$ and $\hat{\boldsymbol{m}}_{\text {dis }}=$ $\left[\hat{m}_{d i s}^{x}, \hat{m}_{d i s}^{y}, \hat{m}_{d i s}^{z}\right]$ are the estimated disturbance forces and moments respectively. $\hat{\boldsymbol{h}}_{\text {dis }}$ can be integrated in (9) and (10)
TABLE I: Mobile base parameters.

\begin{tabular}{|c|c|c|}
\hline Variable & Unit & Explanation \\
\hline \hline$W$ & $\mathrm{~m}$ & Gauge of fixed wheels \\
\hline$R$ & $\mathrm{~m}$ & Radius of fixed wheels \\
\hline$x_{0}, y_{0}$ & $\mathrm{~m}$ & Base center position in the world frame \\
\hline$\psi_{0}$ & $\mathrm{rad}$ & Base orientation \\
\hline$\theta_{r}, \theta_{l}$ & $\mathrm{rad}$ & Rotation angle of the wheels \\
\hline$m$ & $\mathrm{~kg}$ & Total mass of mobile manipulator \\
\hline$I_{W}$ & $\mathrm{kgm}^{2}$ & Inertia of the wheels \\
\hline$I_{\psi}$ & $\mathrm{kgm}^{2}$ & Inertia around the center of the cart \\
\hline $\mathbf{B}_{n}$ & & Nominal inertia matrix \\
\hline $\boldsymbol{K}_{v}$ & & Diagonal velocity gain matrix \\
\hline
\end{tabular}

using the general ZMP formula [9]:

$$
\begin{gathered}
\widehat{Z M P_{x}}=\frac{\Sigma_{i} m_{i} x_{i}\left(\ddot{z}_{i}+g\right)-\Sigma_{i} m_{i} \ddot{x}_{i} z_{i}-\Sigma_{i} I_{i y} \ddot{\theta}_{i y}}{\Sigma_{i} m_{i}\left(\ddot{z}_{i}+g\right)}+\delta_{z m p_{x}}^{d i s} \\
\widehat{Z M P_{y}}=\frac{\Sigma_{i} m_{i} y_{i}\left(\ddot{z}_{i}+g\right)-\Sigma_{i} m_{i} \ddot{y}_{i} z_{i}+\Sigma_{i} I_{i x} \ddot{\theta}_{i x}}{\Sigma_{i} m_{i}\left(\ddot{z}_{i}+g\right)}+\delta_{z m p_{y}}^{d i s} \\
\delta_{z m p_{x}}^{d i s}=\frac{p^{z} \hat{f}_{d i s}^{x}-p^{x} \hat{f}_{d i s}^{z}+{ }^{o} \hat{m}_{d i s}^{y}}{\Sigma_{i} m_{i}\left(\ddot{z}_{i}+g\right)-\hat{f}_{d i s}^{z}} \\
\delta_{z m p_{y}}^{d i s}=\frac{p^{z} \hat{f}_{d i s}^{y}-p^{y} \hat{f}_{d i s}^{z}-{ }^{o} \hat{m}_{d i s}^{x}}{\Sigma_{i} m_{i}\left(\ddot{z}_{i}+g\right)-\hat{f}_{d i s}^{z}}
\end{gathered}
$$

$\boldsymbol{p}=\left[p^{x}, p^{y}, p^{z}\right]$ is the end-effector position. ${ }^{o} \hat{m}_{d i s}^{y}$ and ${ }^{o} \hat{m}_{d i s}^{x}$ are the projection of $\hat{m}_{d i s}^{y}$ and $\hat{m}_{d i s}^{x}$ to origin frame respectively.

\section{Operational Space Position Control of the End-effector}

Position control can be achieved by setting the acceleration command $\boldsymbol{\alpha}_{c}$ in eq.(1) as:

$\boldsymbol{\alpha}_{c}=\ddot{\boldsymbol{r}}_{d}(t)+\boldsymbol{K}_{D r}\left[\dot{\boldsymbol{r}}_{d}(t)-\boldsymbol{v}_{r e s}(t)\right]+\boldsymbol{K}_{P r}\left[\boldsymbol{r}_{d}(t)-\boldsymbol{p}_{r e s}(t)\right]$

$\boldsymbol{v}_{\text {res }}$ and $\boldsymbol{p}_{\text {res }}$ are the velocity and position response of the end-effector computed by the direct kinematics; $\boldsymbol{K}_{D r}$ and $\boldsymbol{K}_{P r}$ are suitable gain matrices; $\ddot{\boldsymbol{r}}_{d}, \dot{\boldsymbol{r}}_{d}(t)$ and $\boldsymbol{r}_{d}(t)$ are the desired acceleration, velocity and position tracking inputs where they are smooth enough for the operational space control in section II-A. In our case they are obtained from the trapezoidal trajectory generator with continuous acceleration as detailed in [43].

\section{Stability Controller}

1) Mobile base velocity controller: The modeling parameters of the mobile base are defined in Table I and depicted in Figure 3. The linear and angular velocities of the base can be calculated as a function of the wheels angular speed as:

$$
\left[\begin{array}{c}
v_{0} \\
\dot{\psi}_{0}
\end{array}\right]=\boldsymbol{J}_{k}\left[\begin{array}{c}
\dot{\theta}_{r} \\
\dot{\theta}_{l}
\end{array}\right]
$$

$\boldsymbol{J}_{k}$ is the kinematic Jacobian defined as:

$$
\boldsymbol{J}_{k}=\left[\begin{array}{cc}
\frac{R}{2} & \frac{R}{2} \\
\frac{R}{W} & -\frac{R}{W}
\end{array}\right]
$$




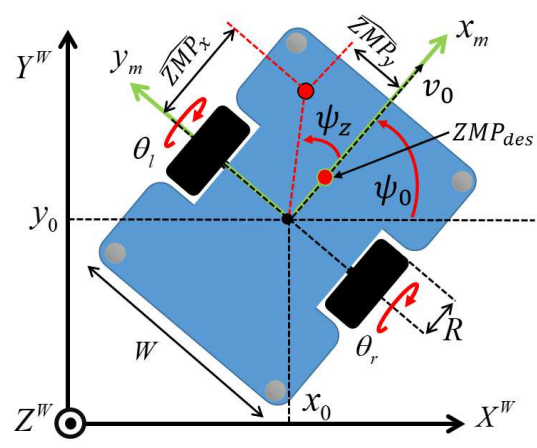

Fig. 3: Mobile base modeling.

Changing the input velocity to the wheels rotational speed gives:

$$
\left[\begin{array}{c}
\dot{\theta}_{r} \\
\dot{\theta}_{l}
\end{array}\right]=\boldsymbol{J}_{k}^{-1}\left[\begin{array}{c}
v_{0} \\
\dot{\psi}_{0}
\end{array}\right]
$$

Consequently, the $2^{\text {nd }}$ order kinematics can be expressed as:

$$
\left[\begin{array}{l}
\ddot{\theta}_{r} \\
\ddot{\theta}_{l}
\end{array}\right]=\boldsymbol{J}_{k}^{-1}\left[\begin{array}{c}
\dot{v}_{0} \\
\ddot{\psi}_{0}
\end{array}\right]
$$

Considering the mass of the whole system, nominal dynamics on the wheels can be written as:

$$
\boldsymbol{\tau}_{w}=\boldsymbol{B}_{n} \ddot{\boldsymbol{\theta}}^{r e f}
$$

$\boldsymbol{\tau}_{w}=\left[\tau_{r}, \tau_{l}\right]^{T}$ is the torque applied on the wheels and $\ddot{\boldsymbol{\theta}}^{r e f}=\left[\ddot{\theta}_{r}^{r e f}, \ddot{\theta}_{l}^{r e f}\right]^{T}$ is the wheels acceleration reference. By applying the Lagrange-Euler formulation to equation (25), the nominal inertia matrix $\boldsymbol{B}_{n}$ can be expressed as:

$$
\boldsymbol{B}_{n}=\left[\begin{array}{cc}
\frac{m R^{2}}{4}+I_{W}+\frac{R^{2}}{W^{2}} I_{\psi} & 0 \\
0 & \frac{m R^{2}}{4}+I_{W}+\frac{R^{2}}{W^{2}} I_{\psi}
\end{array}\right]
$$

The velocity controller of the mobile base can be derived as:

$$
\boldsymbol{\tau}_{w}=\boldsymbol{B}_{n} \ddot{\boldsymbol{\theta}}^{r e f}=\boldsymbol{B}_{n}\left(\boldsymbol{K}_{v}\left(\dot{\boldsymbol{\theta}}^{c m d}-\dot{\boldsymbol{\theta}}^{r e s}\right)+\ddot{\boldsymbol{\theta}}^{c m d}\right)
$$

2) Zero moment point control in the null space: In our approach, the $\widehat{Z M P}$ is expressed in the mobile base frame as shown in Figure 3. Our desired point $Z M P_{\text {des }}$ lies on the $x$-axis of the mobile base $x_{m}$ as it is proven to be the stablest place [9]. For a general arm, with $n \geq 6$ the mobile base can be controlled in the null space and the motions generated by $\boldsymbol{\tau}_{w}$ will fall in $\boldsymbol{\tau}_{\text {null }}$ in eq.(3) by using eq.(6). Hence, stability controller can be designed using the velocity controller presented in Section II-D.1 to maintain the $\widehat{Z M P}$ on $Z M P_{d e s}$ as:

$$
\begin{gathered}
v_{0}^{c m d}=K_{p s}\left(\widehat{Z M P}_{x}-Z M P_{d e s}\right)+K_{d s}\left(C O M_{x}-\widehat{Z M P}_{x}\right) \\
+\dot{\boldsymbol{r}}_{d}^{x}(t) \cos \left(\psi_{0}\right)+\dot{\boldsymbol{r}}_{d}^{y}(t) \sin \left(\psi_{0}\right) \\
\dot{\psi}_{0}^{c m d}=K_{p \psi} \psi_{z}+K_{d \psi}\left(\psi_{z}-\psi_{c}\right) \\
\dot{v}_{0}^{c m d}=\ddot{\boldsymbol{r}}_{d}^{x}(t) \cos \left(\psi_{0}\right)+\ddot{\boldsymbol{r}}_{d}^{y}(t) \sin \left(\psi_{0}\right)
\end{gathered}
$$

with the angles $\psi_{z}$ and $\psi_{c}$ measured from $x_{m}$ to $\widehat{Z M P}$ and the $C O M$ positions in the base frame respectively:

$$
\left\{\begin{array}{l}
\psi_{z}=\arctan 2\left(\widehat{Z M P}_{y}, \widehat{Z M P}_{x}\right) \\
\psi_{c}=\arctan 2\left(\widehat{C O M}_{y}, \widehat{C O M}_{x}\right)
\end{array}\right.
$$

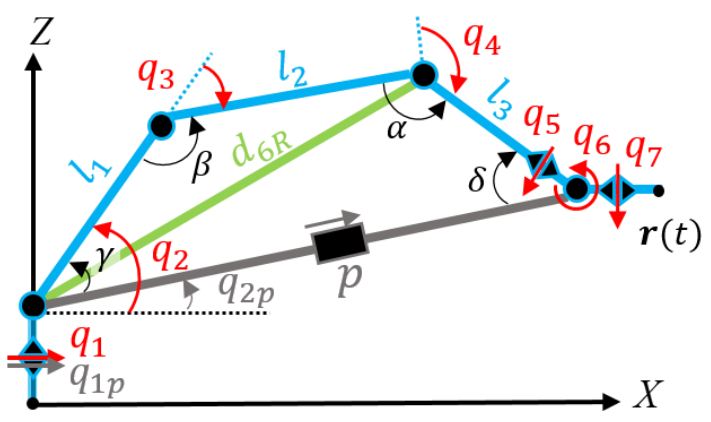

Fig. 4: 7-dof robotic arm kinematics for maximum manipulability.

$$
C O M_{x}=\frac{\sum_{i=1}^{m} m_{i} x_{i}}{\sum_{i=1}^{m} m_{i}}, C O M_{y}=\frac{\sum_{i=1}^{m} m_{i} y_{i}}{\sum_{i=1}^{m} m_{i}}
$$

The adaptive terms $\left(C O M_{x}-\widehat{Z M P}_{x}\right)$ and $\left(\psi_{z}-\psi_{c}\right)$ are used to damp the high dynamic motions of the ZMP. It is acknowledged that the COM is static with low dynamics compared to ZMP. The velocity and acceleration commands of the base in eqs.(28-30) can be applied to the wheels torques $\boldsymbol{\tau}_{w}$ using eqs.(23),(24) and (27). The latter is then changed to the corresponding virtual joint forces of the base as:

$$
\boldsymbol{\tau}_{b}=\boldsymbol{J}_{d} \boldsymbol{\tau}_{w}
$$

\section{E. Manipulability Observer}

When the manipulability of manipulator goes low in the direction of motion, the mobile manipulator can be easily affected by disturbance and its motion becomes unstable. For the redundant arm in Figure 1, we propose manipulability observer to solve redundancy for maximum manipulability. For desired position $\boldsymbol{r}(t)$ the inverse geometric model (IGM) of the prismatic robot $\left(q_{1 p}, q_{2 p}, p\right)$ gives $q_{1 p}^{\max }, q_{2 p}^{\max }$ and $p_{\max }$. For the maximum manipulability $w_{\max }=\max \left(\sqrt{\operatorname{det}\left(\boldsymbol{J}(\boldsymbol{q}) \boldsymbol{J}^{T}(\boldsymbol{q})\right)}\right)$ with respect to the desired position, the quadrilateral formed by connecting $\left(q_{2}, q_{3}, q_{4}, q_{6}\right)$ needs to be with maximum possible area, this can be deduced from [44]. Its inner angles in Figure 4 will be:

$$
\left\{\begin{array}{l}
\alpha=\operatorname{acos}\left(\frac{l_{2}^{2}+l_{3}^{2}-l_{1}^{2}-p_{\max }^{2}}{2\left(l_{2} l_{3}+l_{1} p_{\max }\right)}\right) \\
\delta=\operatorname{acos}\left(\frac{l_{3}^{2} p_{\max }^{2}-l_{2}^{2}-l_{1}^{2}}{2\left(l_{3} p_{\max }+l_{2} l_{1}\right)}\right) \\
\beta=\pi-\delta \\
\gamma=\pi-\alpha
\end{array}\right.
$$

Then,

$$
\begin{gathered}
q_{2}^{\max }=q_{2 p}^{\max }+\gamma-\frac{\pi}{2} \\
q_{3}^{\max }=\beta-\pi \\
q_{4}^{\max }=\alpha-\frac{3 \pi}{2}
\end{gathered}
$$

To maintain the desired orientation, the IGM of the $6 \mathrm{R}$ robot $\left(q_{1}, q_{2}, q_{4}, q_{5}, q_{6}, q_{7}\right)$ gives the desired joint values $q_{5}^{\max }, q_{6}^{\max }$ and $q_{7}^{\max } . d_{6 R}^{\max }$ can be calculated as:

$$
d_{6 R}^{\max }=l_{3}^{2}+p_{\max }^{2}-2 l_{3} p_{\max } \cos \delta
$$


The explicit IGM derivations can be found in [43] and the desired joint values for maximum manipulability are:

$\boldsymbol{q}^{\text {MaxManip }}=\left[q_{1}^{\max }, q_{2}^{\max }, q_{3}^{\max }, q_{4}^{\max }, q_{5}^{\max }, q_{6}^{\max }, q_{7}^{\max }\right]^{T}$

Hence, the arm redundancy can be controlled by means of maximum manipulability observer as:

$$
\boldsymbol{\tau}_{n u l l}^{\text {arm }}=\boldsymbol{P}\left(\boldsymbol{q}^{\text {MaxManip }}-\boldsymbol{q}_{a}\right)+\boldsymbol{D} \frac{d}{d t}\left(\boldsymbol{q}^{\text {MaxManip }}-\boldsymbol{q}_{a}\right)
$$

$\boldsymbol{P}$ and $\boldsymbol{D}$ are proportional and derivative gain matrices respectively.

\section{ADAMS-MATLAB/SIMULINK DYNAMIC SIMULATION}

To test the efficiency of the proposed control, we built our mobile manipulator as shown in Figure 1. The platform consists of a 7-dof arm fixed on a nonholonomic mobile base. The system is simulated using Adams, a simulator with powerful physics engine that allows to study the dynamics of moving parts, and the distribution of forces throughout the mechanical system. The robot dimensions and masses are well respected in comparison to the real platform, friction forces are added to the joints as $0.5 \mathrm{~N} . \mathrm{m}$ for static friction and 0.3 N.m for the dynamic one. The rolling resistance coefficient between the driving wheels and the ground is set to 0.01 . The control strategies discussed above have been successfully implemented in Matlab/Simulink. The Simulink/Adams interface allows bilateral communication. Simulink can send the joint torques to Adams which returns the status of the system (joint positions). The end-effector of the robot is commanded to follow the spatial trajectory $\boldsymbol{r}(t)$ shown in Figure 1 while maintaining its stability. The Cartesian command and response of the trajectory's position and velocity are shown in Figures 5 and 6 respectively. The controller shows good performance, the errors in position are around $0 \mathrm{~mm}$ in constant velocity phases and less than 1 mm during acceleration (Figures 7 and 9), consequently the velocity errors are around zero in the constant phases and maximum of $0.01 \mathrm{~m} / \mathrm{s}$ in the variable phases. No wonder the errors are small as we apply the disturbance observer (DOB) [41] to each joint. In order to ensure the stability of the mobile manipulator while executing the trajectory, the desired zero moment point $Z M P_{\text {des }}$ is set at $0.2 \mathrm{~m}$ on the mobile base axis $x_{m}$ from the base origin (Figure 3). The stability controller presented in Section II-D is able to control the zero moment point around the desired value all along the trajectory, with errors along $x_{m}$ fluctuating around $0 \mathrm{~mm}$ in the constant velocity phases, and less than $5 \mathrm{~mm}$ when the accelerations of the trajectory are on their peak $\left(1 \mathrm{~m} / \mathrm{s}^{2}\right)$ (Figure 9), the ZMP behavior and its errors are shown in Figure 10. Similarly for the orientation, the deviation of the ZMP from $x_{m}$ axis is relatively small, around $0.01 \mathrm{rad}$ as can be seen in Figure 11. The mobile base velocity controller is able to execute the desired velocities $v_{0}^{c m d}$ needed to maintain the ZMP on its desired position, the command and response of the base are plotted in Figure

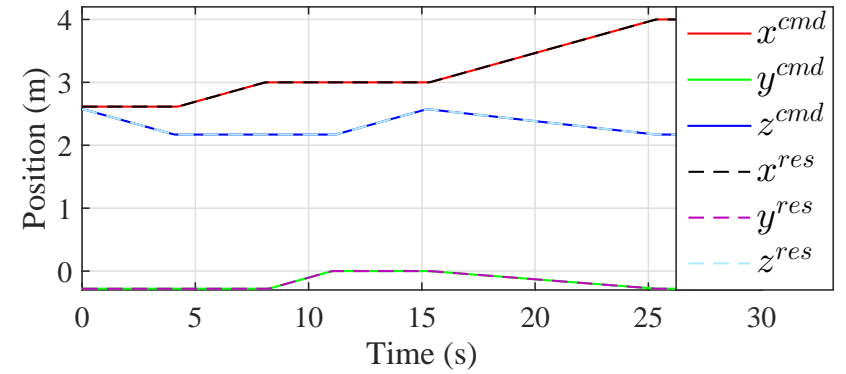

Fig. 5: Position commands vs. responses.

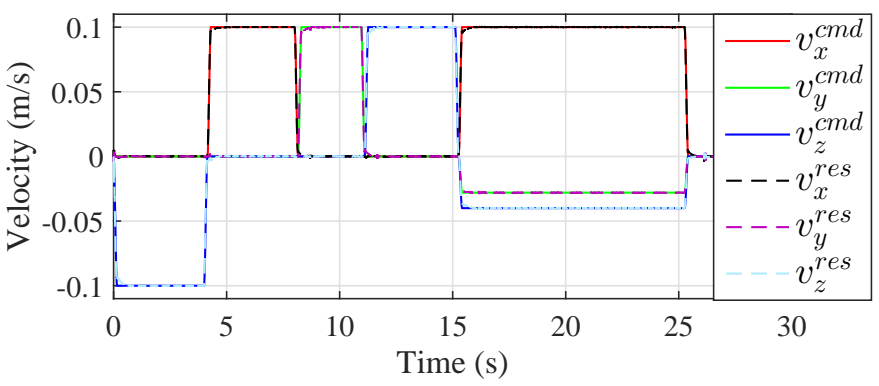

Fig. 6: Velocity commands vs. responses.

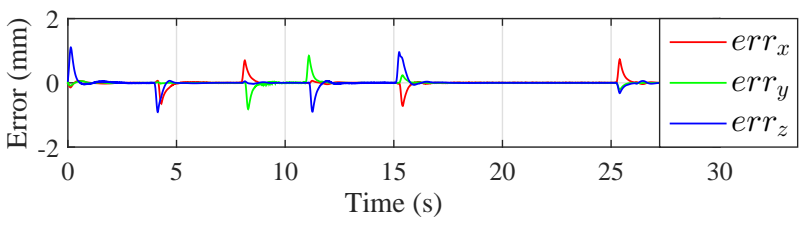

Fig. 7: Position command-response errors.

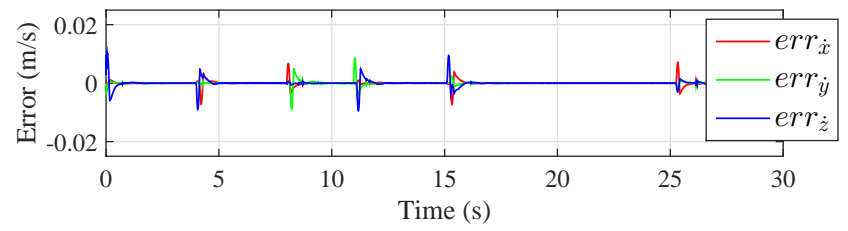

Fig. 8: Velocity command-response errors.

12a, their corresponding errors are shown in Figure 12b. The base velocity is feasible and the execution errors are less than $0.051 \mathrm{~m} / \mathrm{s}$, they are small enough as the ZMP tracking errors are small (Figure 10b). Finally, a top view of the ZMP projection on the base is shown in Figure 13. In this Figure it can be seen that the initial value is driven to the desired one shortly then it stays around $x_{m}=0.2 \mathrm{~m}$ all along the path. Hence, on-line stability control of the mobile manipulator is adequately achieved.

\section{CONCLUSIONS}

This article presents a new approach for controlling the stability of mobile manipulators in real time. The base is controlled in the null space of the task to keep the zero moment point on a desired stable point without affecting the end-effector performance. Additionally, a novel zero moment point observer is presented. The observer estimates the effect of errors in center of mass positions and inertias of the links, 


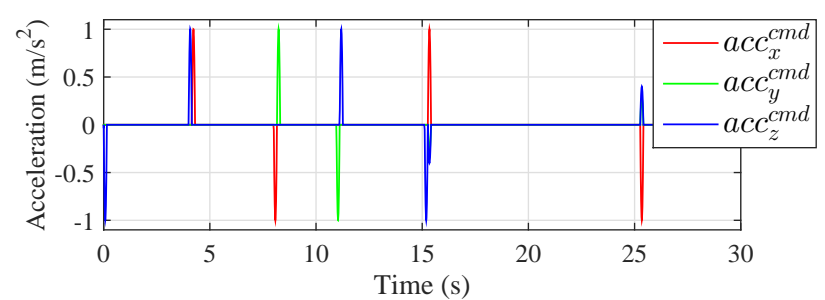

Fig. 9: Acceleration commands.

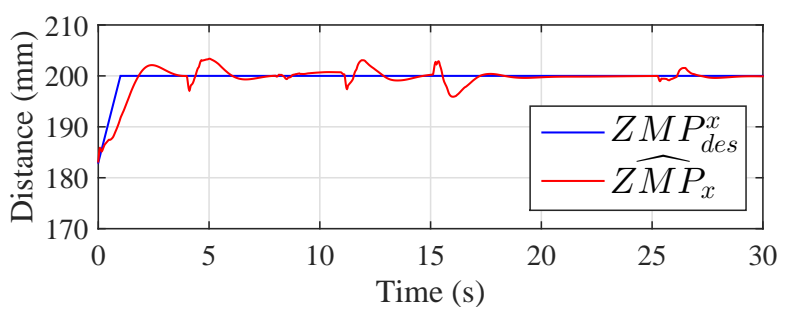

(a) Estimated value $\widehat{Z M P_{x}}$ vs. the desired one $Z M P_{d e s}^{x}$.

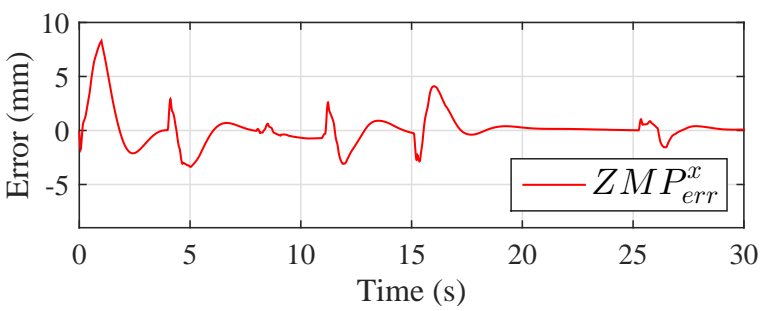

(b) Errors of the ZMP around the desired stable point.

Fig. 10: Zero moment point control on a desired point during the trajectory execution.

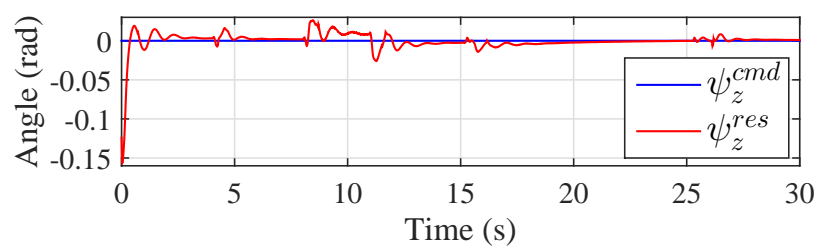

(a) The command vs. response of the ZMP angle from axis $x_{m}$.

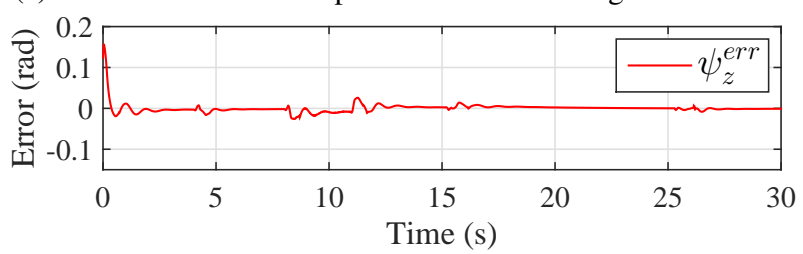

(b) ZMP deviation error from axis $x_{m}$.

Fig. 11: Orientation control of the ZMP deviation from the axis $x_{m}$ (Figure 3 ).

and includes it in the zero moment point position estimation. The controller is validated through dynamic simulations. Not only stability was ensured, but good spatial velocity and position tracking was achieved with very small errors as well. In the near future, the controller will be extended to meet the kinematic limits of the arm then it will be implemented on the real mobile manipulator.

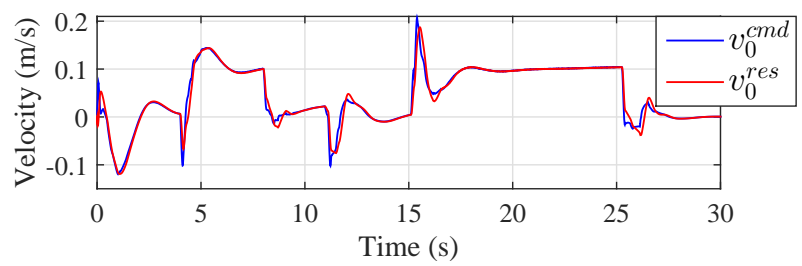

(a) The command vs. response of the mobile base velocity $v_{0}$.

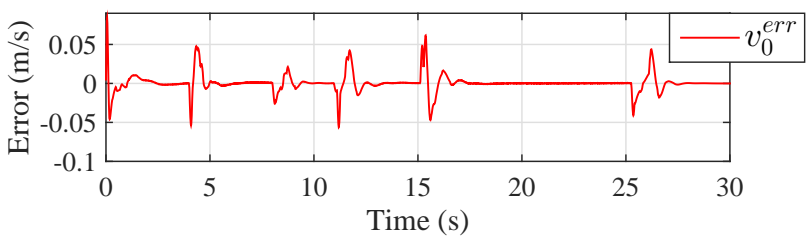

(b) Mobile base velocity errors.

Fig. 12: Orientation control of the ZMP deviation from the axis $x_{m}$ (Figure 3 ).

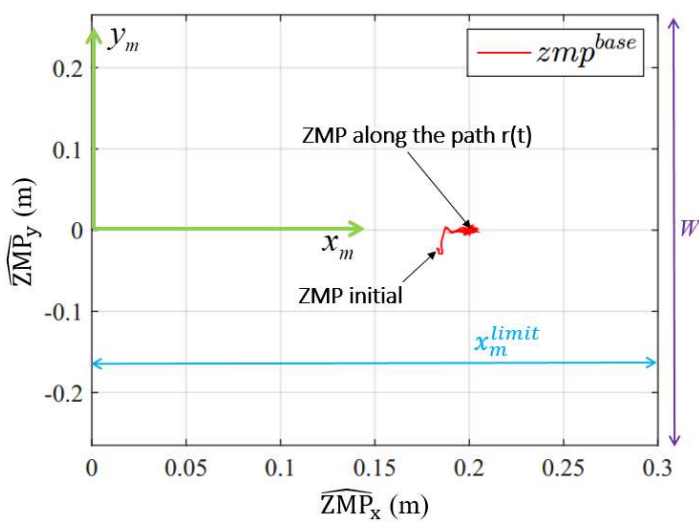

Fig. 13: ZMP projection in the base support plane.

\section{REFERENCES}

[1] "Robots to Re-Construction," http://bots2rec.eu, 2015, [Online; accessed 21-february-2018].

[2] M. Shneier and R. Bostelman, "Literature review of mobile robots for manufacturing," National Institute of Standards and Technology, US Department of Commerce, 2015.

[3] D. E. Orin, R. B. McGhee, and V. Jaswa, "Interactive compute-control of a six-legged robot vehicle with optimization of stability, terrain adaptibility and energy," in Decision and Control including the 15th Symposium on Adaptive Processes, 1976 IEEE Conference on, vol. 15. IEEE, 1976, pp. 382-391.

[4] R. B. McGhee and A. A. Frank, "On the stability properties of quadruped creeping gaits," Mathematical Biosciences, vol. 3, pp. 331351, 1968.

[5] R. B. McGhee and G. I. Iswandhi, "Adaptive locomotion of a multilegged robot over rough terrain," IEEE transactions on systems, man, and cybernetics, vol. 9, no. 4, pp. 176-182, 1979.

[6] I. Hirose, "Umetani: Static stability criterion for walking vehicles," in 21st SICE Symposium, 1978, pp. 253-254.

[7] D. Messuri and C. Klein, "Automatic body regulation for maintaining stability of a legged vehicle during rough-terrain locomotion," IEEE Journal on Robotics and Automation, vol. 1, no. 3, pp. 132-141, 1985.

[8] S. Sreenivasan and B. Wilcox, "Stability and traction control of an actively actuated micro-rover," Journal of Field Robotics, vol. 11, no. 6, pp. 487-502, 1994.

[9] S. Sugano, Q. Huang, and I. Kato, "Stability criteria in controlling mobile robotic systems," in Intelligent Robots and Systems' 93, IROS'93. Proceedings of the 1993 IEEE/RSJ International Conference on, vol. 2. IEEE, 1993, pp. 832-838. 
[10] A. Ghasempoor and N. Sepehri, "A measure of machine stability for moving base manipulators," in Robotics and Automation, 1995. Proceedings., 1995 IEEE International Conference on, vol. 3. IEEE, 1995, pp. 2249-2254.

[11] E. Papadopoulos and D. A. Rey, "A new measure of tipover stability margin for mobile manipulators," in Robotics and Automation, 1996. Proceedings., 1996 IEEE International Conference on, vol. 4. IEEE, 1996, pp. 3111-3116.

[12] K. Yoneda and S. Hirose, "Tumble stability criterion of integrated locomotion and manipulation," in Intelligent Robots and Systems' 96, IROS 96, Proceedings of the 1996 IEEE/RSJ International Conference on, vol. 2. IEEE, 1996, pp. 870-876.

[13] S. A. A. Moosavian and K. Alipour, "On the dynamic tip-over stability of wheeled mobile manipulators," International Journal of Robotics \& Automation, vol. 22, no. 4, p. 322, 2007.

[14] S. Dubowsky and E. Vance, "Planning mobile manipulator motions considering vehicle dynamic stability constraints," in Robotics and Automation, 1989. Proceedings., 1989 IEEE International Conference on. IEEE, 1989, pp. 1271-1276.

[15] W. F. Carriker, P. K. Khosla, and B. H. Krogh, "Path planning for mobile manipulators for multiple task execution," IEEE Transactions on Robotics and Automation, vol. 7, no. 3, pp. 403-408, 1991.

[16] Q. Huang, S. Sugano, and I. Kato, "Stability control for a mobile manipulator using a potential method," in Intelligent Robots and Systems' 94.'Advanced Robotic Systems and the Real World', IROS'94. Proceedings of the IEEE/RSJ/GI International Conference on, vol. 2. IEEE, 1994, pp. 839-846.

[17] Q. Huang and S. Sugano, "Manipulator motion planning for stabilizing a mobile-manipulator," in Intelligent Robots and Systems 95.'Human Robot Interaction and Cooperative Robots', Proceedings. 1995 IEEE/RSJ International Conference on, vol. 3. IEEE, 1995, pp. 467472.

[18] Q. Huang, K. Tanie, and S. Sugano, "Coordinated motion planning for a mobile manipulator considering stability and manipulation," The International Journal of Robotics Research, vol. 19, no. 8, pp. 732$742,2000$.

[19] V. Nunez, N. Nadjar-Gauthier, K. Yokoi, P. Blazevic, and O. Stasse, "Whole body posture controller based on inertial forces," in Humanoid Robots, 2006 6th IEEE-RAS International Conference on. IEEE, 2006, pp. 188-193.

[20] J. Wang, Y. Li, and C. Qiu, "Analysis of dynamic stability constraints for a mobile humanoid robot," in Robotics and Biomimetics, 2008. ROBIO 2008. IEEE International Conference on. IEEE, 2009, pp. $377-382$.

[21] T. Fukuda, Y. Fujisawa, K. Kosuge, F. Arai, E. Muro, H. Hoshino, T. Miyazaki, K. Utubo, and K. Uehara, "Manipulator/vehicle system for man-robot cooperation," in Robotics and Automation, 1992. Proceedings., 1992 IEEE International Conference on. IEEE, 1992, pp. 74-79.

[22] Q. Huang, K. Tanie, and S. Sugano, "Stability compensation of a mobile manipulator by manipulatorpaper motion: feasibility and planning," Advanced Robotics, vol. 13, no. 1, pp. 25-40, 1998.

[23] S. Furuno, M. Yamamoto, and A. Mohri, "Trajectory planning of mobile manipulator with stability considerations," in Robotics and Automation, 2003. Proceedings. ICRA'03. IEEE International Conference on, vol. 3. IEEE, 2003, pp. 3403-3408.

[24] J. Kim and W. K. Chung, "Real-time zero moment point compensation method using null motion for mobile manipulators," Advanced Robotics, vol. 20, no. 5, pp. 581-593, 2006.

[25] D. Choi, M. Kim, and J.-H. Oh, "Development of a rapid mobile robot with a multi-degree-of-freedom inverted pendulum using the modelbased zero-moment point stabilization method," Advanced Robotics, vol. 26, no. 5-6, pp. 515-535, 2012.

[26] S. Lee, M. Leibold, M. Buss, and F. C. Park, "Online stability compensation of mobile manipulators using recursive calculation of zmp gradients," in Robotics and Automation (ICRA), 2012 IEEE International Conference on. IEEE, 2012, pp. 850-855.

[27] Y. Li and Y. Liu, "Real-time tip-over prevention and path following control for redundant nonholonomic mobile modular manipulators via fuzzy and neural-fuzzy approaches," Journal of dynamic systems, measurement, and control, vol. 128, no. 4, pp. 753-764, 2006.

[28] A. Ghaffari, A. Meghdari, D. Naderi, and S. Eslami, "Tipover stability enhancement of wheeled mobile manipulators using an adaptive neurofuzzy inference controller system," in Proceedings of World Academy of Science, Engineering and Technology, vol. 35, no. 241-247, 2008.
[29] L. Yu, Q. Cao, C. Li, and C. Qiu, "On-line planning of nonholonomic mobile manipulators based on stability twist constraint," Journal of the Brazilian Society of Mechanical Sciences and Engineering, vol. 32, no. 2, pp. 165-170, 2010.

[30] K. Liu and F. L. Lewis, "Decentralized continuous robust controller for mobile robots," in Robotics and Automation, 1990. Proceedings., 1990 IEEE International Conference on. IEEE, 1990, pp. 1822-1827.

[31] Y. Yamamoto and X. Yun, "Coordinating locomotion and manipulation of a mobile manipulator," in Decision and Control, 1992., Proceedings of the 31st IEEE Conference on. IEEE, 1992, pp. 2643-2648.

[32] O. Khatib, K. Yokoi, K. Chang, D. Ruspini, R. Holmberg, and A. Casal, "Vehicle/arm coordination and multiple mobile manipulator decentralized cooperation," in Intelligent Robots and Systems' 96, IROS 96, Proceedings of the 1996 IEEE/RSJ International Conference on, vol. 2. IEEE, 1996, pp. 546-553.

[33] J. H. Chung and S. A. Velinsky, "Robust interaction control of a mobile manipulator-dynamic model based coordination," Journal of Intelligent \& Robotic Systems, vol. 26, no. 1, pp. 47-63, 1999.

[34] Y. Yamamoto and X. Yun, "Modeling and compensation of the dynamic interaction of a mobile manipulator," in Robotics and Automation, 1994. Proceedings., 1994 IEEE International Conference on. IEEE, 1994, pp. 2187-2192.

[35] _ - "Effect of the dynamic interaction on coordinated control of mobile manipulators," IEEE Transactions on Robotics and Automation, vol. 12 , no. 5, pp. 816-824, 1996.

[36] W. Miksch and D. Schroeder, "Performance-functional based controller design for a mobile manipulator," in Robotics and Automation, 1992. Proceedings., 1992 IEEE International Conference on. IEEE, 1992, pp. 227-232.

[37] H. Seraji, "A unified approach to motion control of mobile manipulators," The International Journal of Robotics Research, vol. 17, no. 2, pp. 107-118, 1998.

[38] Y. Umeda, D. Nakamura, T. Murakami, and K. Ohnishi, "Hybrid position/force control of a mobile manipulator based on cooperative task sharing," in Industrial Electronics, 1999. ISIE'99. Proceedings of the IEEE International Symposium on, vol. 1. IEEE, 1999, pp. $139-144$.

[39] J. Tan and N. Xi, "Unified model approach for planning and control of mobile manipulators," in Robotics and Automation, 2001. Proceedings 2001 ICRA. IEEE International Conference on, vol. 3. IEEE, 2001, pp. 3145-3152.

[40] O. Khatib, K. Yokoi, K. Chang, D. Ruspini, R. Holmberg, and A. Casal, "Coordination and decentralized cooperation of multiple mobile manipulators," Journal of Field Robotics, vol. 13, no. 11, pp. 755-764, 1996.

[41] K. Ohnishi, "Robust motion control by disturbance observer," Journal of the Robotics Society of Japan, vol. 11, no. 4, pp. 486-493, 1993.

[42] T. Murakami, F. Yu, and K. Ohnishi, "Torque sensorless control in multidegree-of-freedom manipulator," IEEE Transactions on Industrial Electronics, vol. 40, no. 2, pp. 259-265, 1993.

[43] W. Khalil and E. Dombre, Modeling, identification and control of robots. Butterworth-Heinemann, 2004.

[44] T. Yoshikawa, "Manipulability of robotic mechanisms," The international journal of Robotics Research, vol. 4, no. 2, pp. 3-9, 1985. 